\title{
Is percutaneous cholecystostomy a safe alternative for neutropenic patients with severe biliary septic shock?
}

\author{
Nicolae I Simion
}

\begin{abstract}
Introduction: Acalculous cholecystitis is a relatively rare form of acute cholecystitis appearing in critically ill patients. Case Report: We present the case of a male patient under chemotherapy for myeloid leukemia who developed a severe septic shock secondary to an acute acalculous cholecystitis. Because of hemodynamic instability requiring high-dose of vasoactive amines, percutaneous gallbladder drainage was done. After this intervention, the septic shock could be controlled, but a bleeding liver laceration was observed, fortunately without morbidity consequences. Conclusion: Acute acalculous cholecystitis is a lifethreatening condition in neutropenic patients and percutaneous gallbladder drainage could be a minimally invasive and safe alternative treatment in high-risk patient.
\end{abstract}

Keywords: Alithiasic cholecystitis, Thrombopenia, Neutropenia, Septic shock, Cholecystostomy, Gallbladder drainage

$* * * * * * * * *$

Nicolae I Simion

Affiliations: Riviera Hospital, General Surgery Department, Avenue Belmont no. 25, 1820 Montreux, Switzerland.

Corresponding Author: Nicolae I Simion, M.D. Riviera Hospital, General Surgery Department, Avenue Belmont no. 25, 1820 Montreux, Switzerland; Ph: +41219666736; +41 764945008; Email: nicolaeirinel.simion@hopitalriviera.ch; irisnicol@gmail.com

Received: 12 December 2011

Accepted: 06 January 2012

Published: 31 January 2012
Simion NI. Is percutaneous cholecystostomy a safe alternative for neutropenic patients with severe biliary septic shock? International Journal of Hepatobiliary and Pancreatic Diseases 2012;2:1-3.

Article ID: 100003IJHPDNIS2012

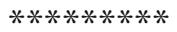

doi:10.5348/ijhpd-2012-3-CR-1

\section{INTRODUCTION}

Acalculous cholecystitis is a form of acute cholecystitis seen more frequently in immunosuppressed and critically ill patients, typically in ICU units. The diagnosis and management of this condition is a real challenge for the physician, because of the lack of resources in this patient population.

\section{CASE REPORT}

We report the case of a 36-year-old man with the diagnosis of acute myeloid leukemia type M4. The patient was under cytoreduction chemotherapy followed by induction chemotherapy. During this period the patient was admitted in hospital with diarrhea and fever. The laboratory tests showed a low total leukocyte count of $850 / \mathrm{mm}^{3}$ and hemoglobin level of $8.9 \mathrm{~g} / \mathrm{dl}$. Neutropenic colitis was considered on clinical basis and confirmed by computed tomography (CT) of the abdomen. The patient was treated by $4.5 \mathrm{gm}$ piperacillin/tazobactam, administered three times a day, with consecutive good recover. Colonoscopy and biopsy of the colon were not considered necessary for diagnosis.

One month later the first consolidation chemotherapy was done and after six days the patient was again admitted with diarrhea. The laboratory 
investigations revealed a low total leukocyte count of $500 / \mathrm{mm}^{3}$, with neutrophils - $36 \%$, lymphocytes - $32 \%$, eosinophils - 6\%, monocytes - 20\% and basophils - $6 \%$. The hemoglobin level was $6.8 \mathrm{~g} / \mathrm{dl}$ and platelet count was $11000 / \mathrm{mm}^{3}$. Despite antibiotics for a supposed recidivating colitis $(1500 \mathrm{mg} /$ day imipenem divided in three doses) and supportive treatment, the patient's clinical status degraded. Patient developed right upper quadrant abdominal pain, high fever $\left(40^{\circ} \mathrm{C}\right)$ and hypotension requiring ICU admission with a diagnosis of septic shock of abdominal origin. A positive Murphy's sign was observed on abdominal examination. Radiological imaging (CT scan) was suggestive of acalculous cholecystitis and also showed transverse colon wall thickening (figure 1). Antibiotics were changed to $13.5 \mathrm{~g} /$ day, piperacillin/tazobactam divided in three doses.

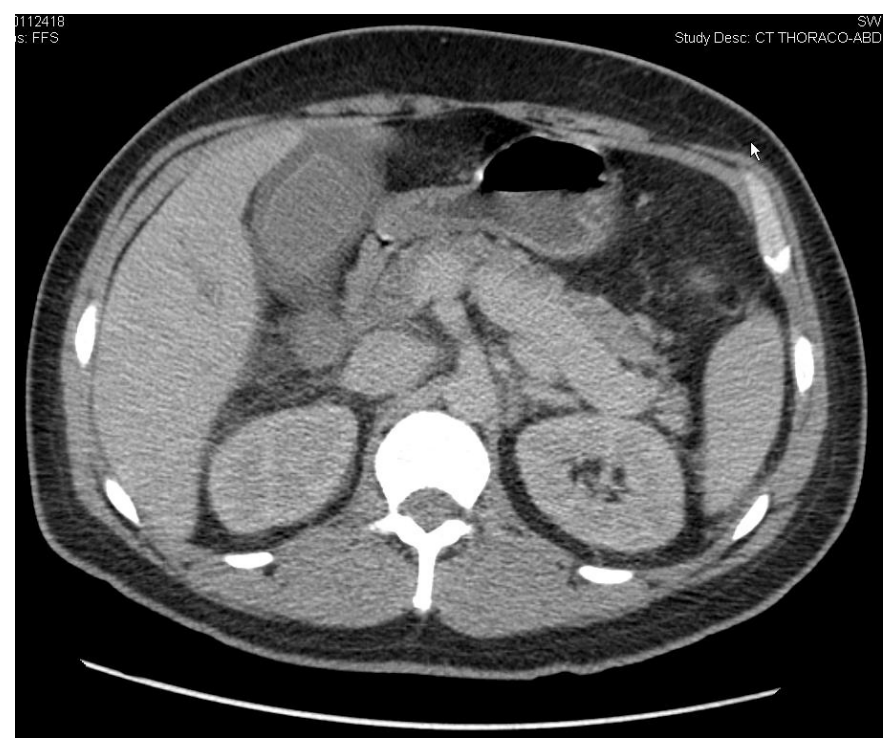

Figure 1: Abdominal CT scan demonstrating gallbladder wall thickening and pericholecystic liquid, without gallbladder lithiasis, confirmed by ultrasonography.

The patient needed high doses of vasoactive therapy because of severe hypotension secondary to septic shock. He was given a continuous intravenous infusion of vasopressin at 0.04 units/min and $50-120 \mu \mathrm{g} / \mathrm{min}$ of norepinephrine. He was considered to unstable for a surgical intervention and despite his low platelet count of $7000 / \mathrm{mm}^{3}$, percutaneous CT-guided gallbladder drainage was done. The patient received five units of platelet transfusion but the platelet count failed to increase above $15000 / \mathrm{mm}^{3}$. The septic shock rapidly improved in less than 24 hours after this procedure. The microbiological culture of bile sample was negative for bacteria. The next day hemoglobin level fell from 8.3 to $6.1 \mathrm{~g} / \mathrm{dl}$. A CT scan revealed a bleeding liver laceration with intraperitoneal hemorrhage after the transhepatic percutaneous gallbladder drainage (figure 2).

The patient was treated conservatively receiving three units of red blood cells (RBC) concentrate and one unit of fresh frozen plasma. Antibiotic therapy with

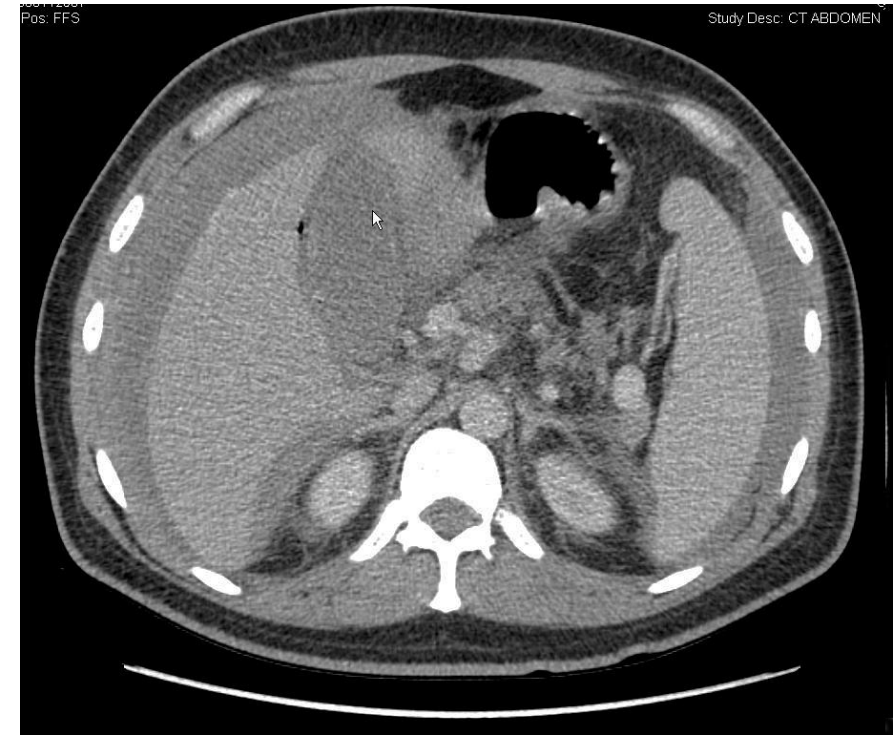

Figure 2: Abdominal CT scan showing peritoneal bleeding from hepatic laceration after gallbladder percutaneous transhepatic drainage.

piperacillin/tazobactam as described above and 1000 mg amikacin divided in two doses was continued. He remained in the hospital for 10 days after gallbladder drainage, going home well with no further complications.

\section{DISCUSSION}

Patients with lymphoma or leukemia undergoing aggressive chemotherapy are often affected by abdominal infections which become life-threatening conditions. It is a clinical diagnostic challenge for the physician to find the cause of abdominal symptoms (infectious and non-infectious) in these patients $[1,2]$.

Abdominal infections are life-threatening complications in neutropenic patients. Neutropenic enterocolitis is the most important and common entity, but acute cholecystitis is rare and has been described in several reports, suggesting that this infection could represent a difficult trap [1].

Acalculous cholecystitis is an entity occurring typically in elderly, severely ill or high risk patients hospitalized in the ICU. The frequency of acute cholecystitis in patients with myelosupressive chemotherapy and neutropenia is estimated at $0.4 \%$ in literature, with a nearly $30 \%$ mortality. The majority of the neutropenic patients develop acalculous cholecystitis, in contrast with non-neutropenic patients to whom acalculous cholecystitis comprises only approximately $5 \%$ of all cases of acute cholecystitis [1].

The clinical diagnosis of acute cholecystitis (AC) remains difficult in the patient population with multiples comorbid conditions, the typical signs and symptoms being easily masked in ventilated, paralyzed or immunosuppressed patients. Abdominal 
ultrasonography is an established valuable imaging method if cholecystitis is suspected and a thickened gallbladder wall greater than $3 \mathrm{~mm}$ is considered typical for diagnosis. Other frequent sonographic findings are pericholecystic fluid and overdistension. Similar findings can be demonstrated by CT scan. Computed tomography is extremely useful in the assessment of septic shock of unknown origin in this patient population $[1,3]$.

Laparoscopic cholecystectomy is widely considered as the treatment of choice for acute cholecystitis and it can be performed safely in selected patients. Surgery in patients with neutropenia, thrombocytopenia and sepsis who present with an acute abdomen is however associated with a high mortality. Percutaneous cholecystostomy (PC) was described as a minimally invasive, image-guided procedure and has become an alternative treatment strategy for high-risk patients with acute calculous or acalculous cholecystitis who are unfit for urgent surgery $[1,4]$.

Percutaneous cholecystostomy could be also used as a method of exclusion of the gallbladder/biliary tree as a source of sepsis in unstable severely ill patients. The positive response is defined as resolution of right upper quadrant abdominal pain and normalization of general clinical state, usually within 48 hours [3].

The procedure can be performed under CT or USguidance, even at the bedside in the ICU. The safety of the procedure and its minimal invasiveness made it a valid treatment option for patient not responding to antibiotic therapy or being in severe shock and unfit for immediate cholecystectomy. The results from several series prove that the percutaneous gallbladder drainage is safe and that it was effective in more than $85 \%$ of patients. There were no bile duct injuries or any other major surgical complications in this group of patients [5].

One of the particularities of our report was the rapid development of a profound septic shock not responding to large spectrum antibiotic therapy; this could be explained by severe immunodeficiency secondary to previous aggressive chemotherapy. Another point was the importance of clinical diagnosis that pointed us towards a biliary pathology and despite no bacteria identification in the bile sample, the patient's state improved dramatically after the gallbladder drainage. This procedure was also difficult to choose, because of patient's thrombocytopenia and because of the uncertain origin of sepsis. However, considering that the patient's hemodynamic state was not stable enough for a surgical intervention, the percutoneous drainage was preferred to the strategy of wait and watch. The hemorrhagic complication showed afterwards the thin margin that we had between right and wrong in a difficult situation.

\section{CONCLUSION}

Though rare, acute acalculous cholecystitis is a lifethreatening condition in neutropenic patients and percutaneous gallbladder drainage could be a minimally invasive and safe alternative treatment in high-risk patient.

$$
* * * * * * * * *
$$

\section{Acknowledgements}

Professor Nicolas Demartines, Visceral surgery, University Hospital Lausanne - CHUV, Switzerland

\section{Author Contributions}

Nicolae I Simion - Conception and design, acquisition of data, analysis and interpretation of data; Drafting the article and revising it critically for important intellectual content; Final approval of the version to be published

\section{Guarantor}

The corresponding author is the guarantor of submission.

\section{Conflict of Interest}

We do not have any financial interest of interest

\section{Copyright}

(C) Nicolae I Simion 2012; This article is distributed under the terms of Creative Commons attribution 3.0 License which permits unrestricted use, distribution and reproduction in any means provided the original authors and original publisher are properly credited. (Please see www.ijhpd.com/copyright-policy.php for more information.)

\section{REFERENCES}

1. Gorschlüter M, Mey U, Strehl J, et al. Cholecystitis in neutropenic patients: Retrospective study and systematic review. Leukemia Research 2006;30:5218.

2. Hatzidakis AA, Prassopoulos P, Petinarakis I, et al. Acute cholecystitis in high-risk patients: percutaneous cholecystostomy vs conservative treatment. Eur Radiol 2002;12:1778-84.

3. Beardsley SL, Shlansky-Goldberg RD, Patel A, et al. Predicting Infected Bile among patients undergoing percutaneous cholecystostomy. Cardiovasc Intervent Radiol 2005;28:319-25.

4. Silberfein EJ, Zhou W, Kougias P, et al. Percutaneous cholecystostomy for acute cholecystitis in high-risk patients: experience of a surgeoninitiated interventional program. The American Journal of Surgery 2007;194:672-7.

5. Barie PS, Eachempati SR Acute acalculous cholecystitis. Curr Gastroenterol Rep 2003 Aug;5(4):302-9. 